\title{
Failure mechanisms in alloy of polyamide 6,6/polyphenylene oxide under severe conditions
}

\author{
H. J. SUE*, A. F. YEE \\ Macromolecular Research Center and Department of Materials Science and Engineering, \\ University of Michigan, Ann Arbor, MI 48109, USA
}

Toughening mechanisms of a polyamide 6,6/polyphenylene oxide alloy containing an elastomer tested under a slow rate, an impact rate, and a low temperature have been investigated using various microscopy techniques. It is found that the toughening mechanisms of the alloy may change from crazing/shear yielding, to crack bridging/crazing, and to transparticle failure, depending on the testing conditions. Except for the low temperature high strain rate testing condition and in the plane stress region of the crack, the crazing mechanism has been observed in all the conditions we studied. When the testing rate is high, the shear yielding mechanism is suppressed; multiple crazing and particle bridging mechanisms appear to dominate.

\section{Introduction}

Most of the engineering polymers are either brittle or notch sensitive [1]. This is especially true with multiphase polymer alloys. They can sometimes be modified to possess high toughness at room temperature and at relatively low deformation rates [2-5]. The toughening effect, however, could drop dramatically when these toughened polymers are deformed at low temperatures or at high strain rates, i.e., the polymer could go through a ductile-brittle transition. The cause for such disappointing results may be that at low temperatures and high testing rates, the efficient energy absorbing mechanisms such as crazing and shear banding may not be able to operate due to the viscoelastic and viscoplastic nature of polymers. It is also possible that the low temperature approaches the $T_{\mathrm{g}}$ of the rubber toughener. Consequently, brittie failure ensues.

A quantitative description of the nature of ductilebrittle transition of polymers requires understanding the physical nature of polymer relaxation behaviour as well as careful examination of all the possible operative toughening mechanisms at the given geometry, temperature, and rate conditions. The present work focuses on the deformation mechansims of the polyamide 6,6/poly 2,6-dimethyl 1,4-phenylene oxide (PA/PPO) system, under a slow rate, an impact testing rate, and low temperature conditions.

Fourteen available toughening mechanisms have been reported in polymer alloys when fracture occurs [6]. Among these, some may co-exist simultaneously or sequentially around the crack tip, and may even interact, depending on the nature of the polymer and the corresponding testing conditions [2-12]. Some of the mechanisms, such as crack bridging, crack deflection, and crack pinning, tend to prevail as the material becomes more brittle. The crack tip blunting and plastic flow mechanisms tend to occur only when shear yielding can occur easily. Each type of toughening mechanism has its optimal conditions for operation. It is thus imperative that one critically examines the failure mechanisms of the material under specific testing conditions in order to effectively toughen the material for specific needs.

We have previously shown that dilatational types of mechanisms such as crazing, debonding, and internal cavitation of the toughener phase have to be activated to allow the occurrence of the shear banding mechanism when the sample is tested under constrained conditions $[2-5,7-9]$. We have also found that the crack bridging and shear yielding mechanisms do not co-exist in the slow crack growth zone in any of the toughened polymer alloys we studied [3-5, 7-9].

The current study is a continuation of our previous effort [5] which mainly discusses the toughening mechanisms of PA/PPO alloy in the plane strain slow crack growth regime. The deformation mechanisms in the fast fracture region (see Fig. 1a), at low temperatures, and at high strain rates have not been extensively investigated in the literature. We hope that this study can provide useful insight concerning the toughening of polymers under these conditions. Based on the above understanding, methodology for improving impact properties and low temperature behaviour of polymers can be sought. Further, the understanding of the fast fracture deformation mechanisms

* Present address: B-1470, Texas Polymer Center, Dow Chemical USA, Freeport, TX 77541, USA. 
Fast fracture region Razor tapping

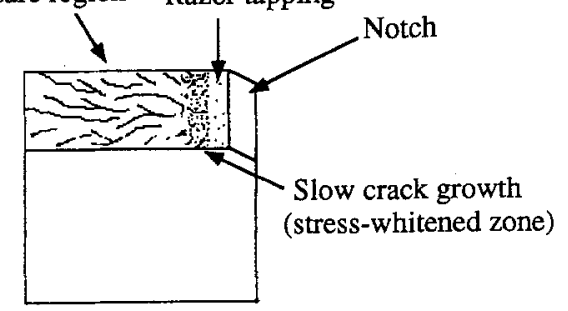

(a)

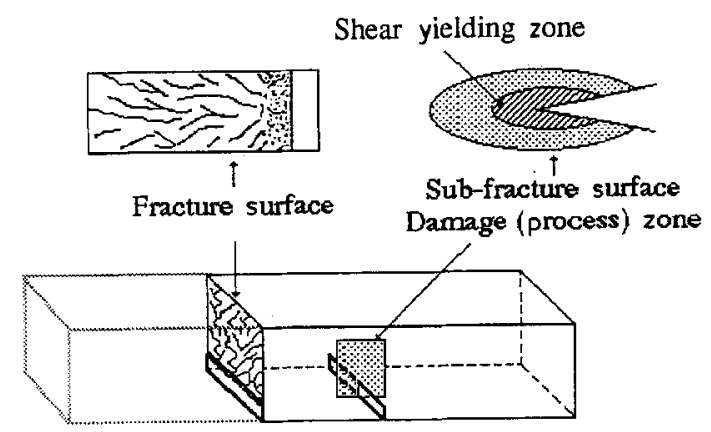

(b)

Figure 1 A schematic drawing of (a) the SEN-3PB fracture surface and (b) the damage zone of the DN-4PB specimen.

of the PA/PPO alloy may help provide insight into the toughening of inherently brittle polymers, such as highly crosslinked thermosets.

Another important factor that needs to be considered is the stress field the material experiences when tested. For a specimen containing a sharp crack but a relatively small thickness, or a blunt crack, or at the surface of a thick specimen, the material would experience the plane stress condition. On the other hand, for the plane strain condition to prevail, the specimen needs to be thick and must possess a sharp crack in it. In other words, the stress field the material experiences depends greatly on the specimen geometry. As a result, one would expect different operating failure mechanism(s) for different specimen geometries. Alternative toughening strategies must therefore be implemented for different dominating stress states $[2,6,9]$.

There have been disputes over the sequence of toughening events and the major causes for shear yielding in front of the crack tip $[2,5,10,14-17]$. It is mainly due to the inappropriate extrapolation of observations from the plane stress condition to the plane strain condition. The main cause for the observed sequence of toughening events and localized shear yielding around the propagating crack in the plane stress region can be very different from that in the plane strain region. Hence, the present work will also investigate how the failure process is affected by the stress state the material experiences.

The fracture and sub-fracture surface regions of the tested single-edge-notch three-point-bend (SEN-3PB) specimens at various conditions have been tested and studied using scanning electron microscopy (SEM), transmitted light optical microscopy (TOM) and transmission electron microscopy (TEM) techniques.
The damage zones observed both at the plane strain and the plane stress regions are compared using the double-notch four-point-bend (DN-4PB) technique $[5,13]$ (Fig. 1b).

\section{Experimental procedure}

The PA/PPO alloy, known commercially as GTX ${ }^{\circledR}$ 910, was obtained from the General Electric Company. According to the manufacturer's literature, this material is comprised of $50 \%$ by weight PA and $50 \%$ by weight PPO. The latter is in turn modified with an average of $15 \%$ of the elastomeric material. Details of the sample preparation have been reported elsewhere [5]. Only a simple description will be given here.

The SEN-3PB, DN-4PB and Izod impact specimens were prepared from injection moulded bars of dimensions $63.5 \mathrm{~mm} \times 12.7 \mathrm{~mm} \times 6.35 \mathrm{~mm}$. The starter crack was made by first cutting with a diamond saw to give an appropriate notch length and then by wedging open a crack with a fresh razor blade which had been cooled in liquid nitrogen. This procedure ensures that a sharp crack tip is created. In the case of the double-notched specimen efforts were made to ensure that the cracks thus generated were as nearly equal in length as possible. For investigations on the fast fracture region (Fig. 1), the SEN-3PB specimens were tested at $8.47 \mathrm{~mm} \mathrm{~s}^{-1}$ using a screw driven tensile testing machine (Instron model 1137). The high rate experiment was conducted using a Sonnpat Universal Impact Tester (Model SI-1) at a rate of $\approx 4 \mathrm{~m} \mathrm{~s}^{-1}$.

The low temperature SEN-3PB specimen was first immersed into a liquid nitrogen bath until equilibrated and quickly removed for Izod impact test with the conditions described above.

The DN-4PB specimens were tested at $8.47 \mathrm{~mm} \mathrm{~s}^{-1}$ using a screw driven testing machine (Instron model 1137). During the test, care was taken to ensure that the two upper loading points contacted the specimen simultaneously while the specimen was supported at the outer loading points.

After fracturing, thin, $30 \mu \mathrm{m}$ films of PA/PPO were obtained by polishing, following the procedure described by Holik, et al. [18]. Sections were taken from the mid-plane (plane strain region) of the fractured SEN-3PB and both the mid-plane and skin (plane stress region) of the survived crack of the DN-4PB specimens (Fig. 1b). The sections were made normal to the fracture surface and parallel to the cracking direction. When the thin film of the skin region was prepared, caré was taken to ensure that a flat surface was obtained by using 1200 grit polishing paper. It was then followed by buffing with $0.3 \mu \mathrm{m}$ alumina powder in a water suspension to avoid penetration of the alumina into the depth of the specimen. The thin films were then studied using a Nikon Microphot optical microscope.

The fracture surface of the SEN-3PB specimen was coated with a $20 \mathrm{~nm}$ layer of Au-Pd for SEM (Hitachi S-520) studies. The sub-fracture surface zone was studied by cutting a proper block size in a section normal to the fracture surface and parallel to the cracking direction. They were then stained with a $2 \%$ 
aqueous solution of $\mathrm{OsO}_{4}$ for about $48 \mathrm{~h}$. Afterwards, these blocks were microtomed using a Reichert Ultracut $\mathrm{E} 43$ to make thin sections on the order of $0.1 \mu \mathrm{m}$ in thickness. The thin sections were laid on top of copper grids and examined using a Zeiss EM-10A (or Philips 400) TEM operating at an accelerating voltage of $100 \mathrm{kV}$.

\section{Results}

Since the present study mainly focuses on the brittle fracture mechanisms of PA/PPO alloy, the failure mechanisms in the unstable fracture regions will be discussed. The failure mechanisms of this alloy in the plane strain slow fracture region (stress-whitened zone) can be found in a previous paper [5]. Failure mechanisms both at the plane strain and the plane stress regions are compared and discussed, as well.

Figure 2 is a SEM micrograph taken in the fast fracture region of the fracture surface of the SEN-3PB sample which had been tested at room temperature and at a crosshead speed of $8.47 \mathrm{~mm} \mathrm{~s}^{-1}$. The dispersed phase of PPO particles is lighter in appearance. These particles appear to have been elongated, which signifies the occurrence of the particle bridging mechanism when the crack propagates along in the fast fracture region. Strong adherent strength at the interface, which leads to the drawing of the PPO particles, is evident. However, adhesive failure can also be observed. In order to confirm the above observations, TEM investigations were conducted.

In the sub-fracture surface zone (SFSZ), as shown in Fig. 3 (using TEM), the elongation of the PPO particles is in a direction approximately perpendicular to the fracture surface. This type of deformation is very different from that in the stress-whitened zone created by the relatively slow, stable crack growth, where the PPO particles appear to have shear yielded at an angle to the fracture surface (see Fig. 11a in [5]). It is noted that the drawing of the PPO particles is ob-

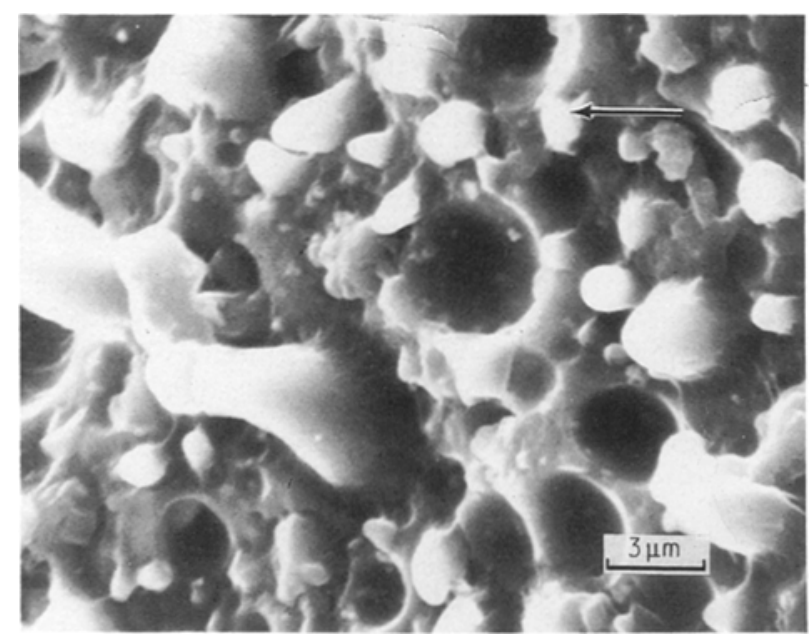

Figure 2 SEM micrograph of the SEN-3PB specimen taken in the fast fracture region at a crosshead speed of $8.47 \mathrm{~mm} \mathrm{~s}^{-1}$. The lighter-coloured dispersed phase is PPO. The PPO particles have been highly elongated when the crack propagated through the matrix surrounding them. The arrow indicates the crack propagation direction

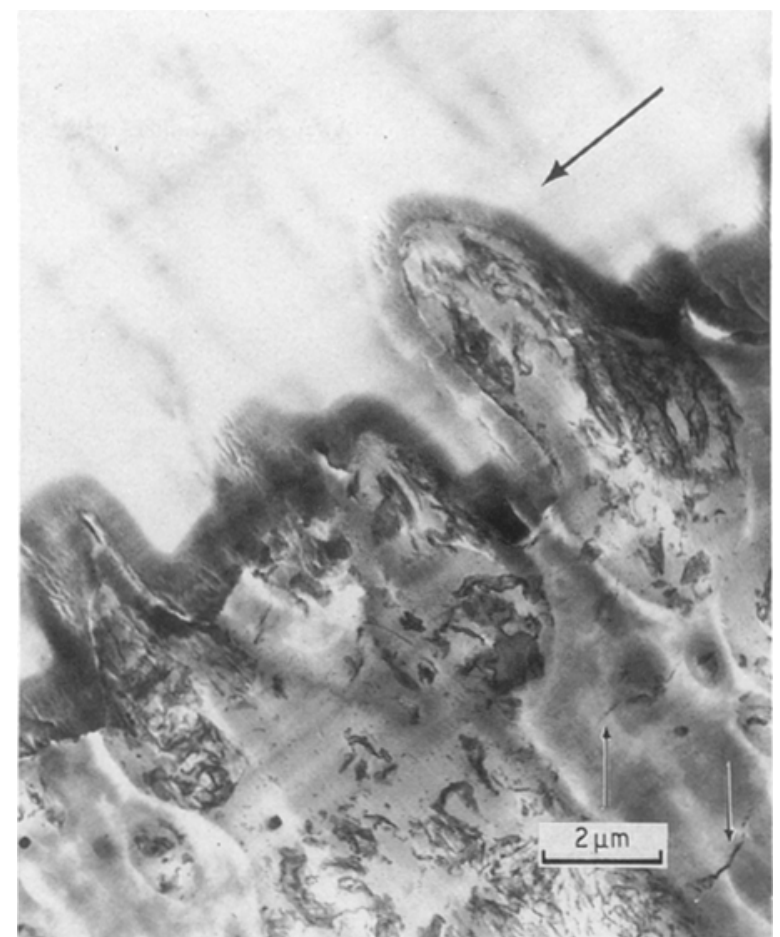

Figure 3 TEM micrograph taken at the SFSZ of the fast fracture region at a testing rate of $8.47 \mathrm{~mm} \mathrm{~s}^{-1}$. The $P P O$ particles have been drawn perpendicular to the fracture surface, similar to that in Fig. 2. Scattered crazes, as indicated by the small arrows, can also be seen immediately beneath the crack face. The big arrow indicates the crack propagation direction.

served only on the crack face. The particles beneath the crack face remain relatively undeformed, i.e., the PPO particles stay approximately spherical. This is also very different from the case of the PPO particles in the plane strain stress-whitened zone [5]. Scattered crazes, which appear to be initiated from the rubbery phase inside the PPO particles, are also seen in the SFSZ in the PA matrix. Since these crazes are very fine and are confined immediately beneath the fracture surface, they cannot be resolved by TOM (Fig. 4).

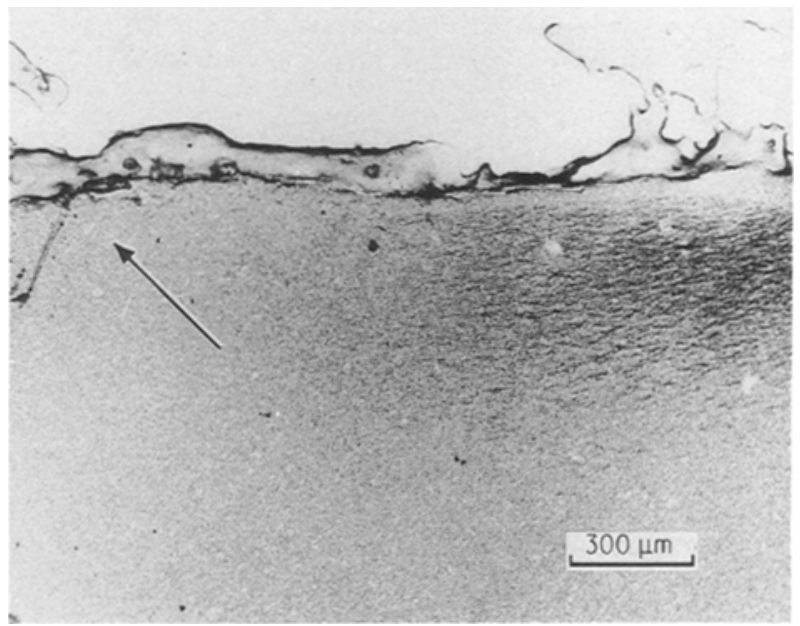

Figure 4 A transmitted light optical micrograph taken at the SFSZ of the SEN-3PB specimen fractured at a testing rate of $8.47 \mathrm{~mm} \mathrm{~s}^{-1}$. The craze pattern can no longer be observed in the fast fracture region, as indicated by the arrow. The crack propagates from right to left. 

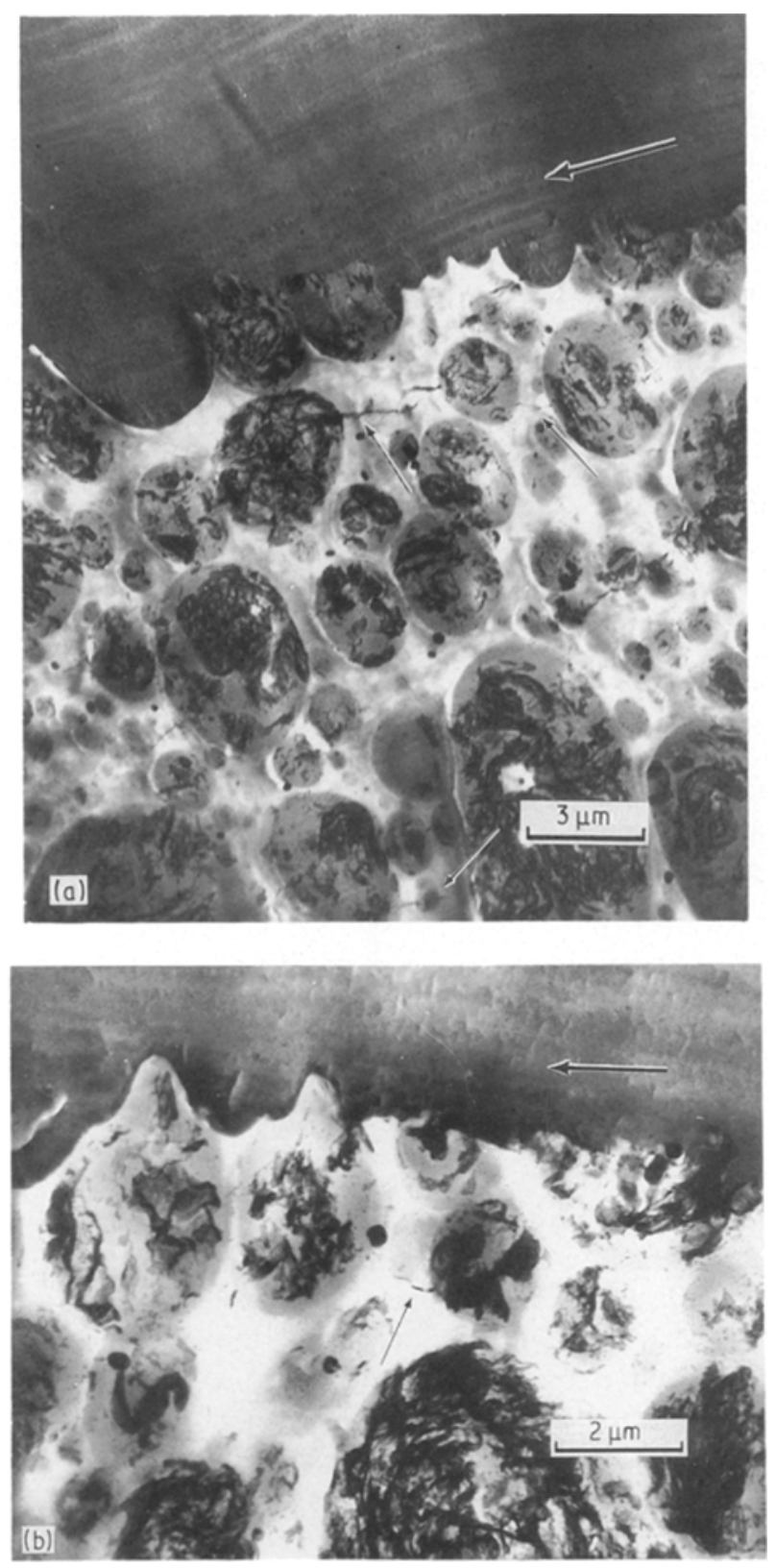

Figure 5 TEM micrographs taken at the SFSZ of the impact-tested $\left(4 \mathrm{~m} \mathrm{~s}^{-1}\right)$ specimen. Both (a) and (b) are taken at the SFSZ. The PPO particles have been elongated moderately, which is similar to the micrograph shown in Fig. 3. Scattered crazes, as indicated by the small arrows, are also observed immediately beneath the crack face. The big arrow indicates the crack propagation direction.

Figure 4 is. a TOM micrograph taken at the SFSZ of the SEN-3PB specimen. It is evident that the crazed zone, which surrounds the shear yielded zone, forms in front of the crack tip (see also Figs 6 and 10 in [5]). Ahead of the crazed zone, no resolvable feature is seen using TOM. Therefore, TOM investigations will not be used to study the failure mechanisms of PA/PPO under impact and low temperature tests.

In the case of the deformation mechanisms at a high testing rate, i.e., $4 \mathrm{~m} \mathrm{~s}^{-1}$, little or no plastic deformation is apparent from TOM of the SFSZ or by SEM on the fracture surface. However, when TEM is used, limited plastic drawing of the PPO particle can still be observed (Fig. 5). A few thin crazes are also found in

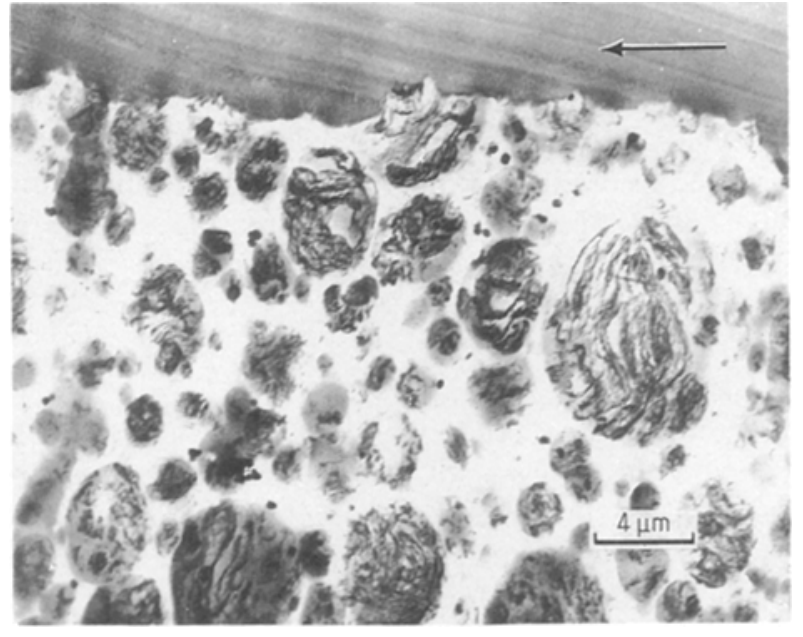

Figure 6 TEM micrograph taken at the SFSZ of the low temperature and impact-tested specimen. Transparticle fracture is found at the crack face. Interfacial adhesive failure is also observed. The arrow indicates the crack propagation direction.

the SFSZ. This feature is essentially similar to that of the SFSZ of the fast fracture region shown earlier (Fig. 3) except for the lower degree of drawing of the PPO particles. Interfacial adherent failure is also observed.

For the specimen tested after immersion in liquid nitrogen and at an impact rate $\left(\approx 4 \mathrm{~m} \mathrm{~s}^{-1}\right)$, no significant toughening mechanisms are operative. Transparticle fracture of the PPO phase as well as interfacial failure are found (Fig. 6). No crazes are observed in this case though the fracture may have been preceded by a few crazes.

In order to compare the failure mechanisms between the plane strain and plane stress regions, the subcritically propagated crack of the DN-4PB specimen was studied both in the skin and in the core regions. As shown in Figs 7 and 8, the damage zones between the two regions are quite different. When viewed in bright field (Fig. 7), it is apparent that the crazing mechanism appears to be inoperative in the plane stress surface region. By contrast, a rather large crazed zone is found in the plane strain region (see [5] for the verification of the crazed zone). When the damage zones are viewed under crossed-polars, the sizes of the birefringent shear yielded zones between the two regions are similar. The shear yielded zone at the plane strain region is encompassed by the crazed zone, which was first observed in [5]. A careful contour mapping of the two shear yielded zones shows that, in fact, the plane strain shear yielded zone is slightly larger than that in the plane stress region. These observations imply that the cavitation process, which is evinced by the crazed zone, does play an important role in inducing shear yielding in the plane strain region.

\section{Discussion}

The purpose of the present study is to gain insight into the toughening mechanisms in the multi-phase rigid- 

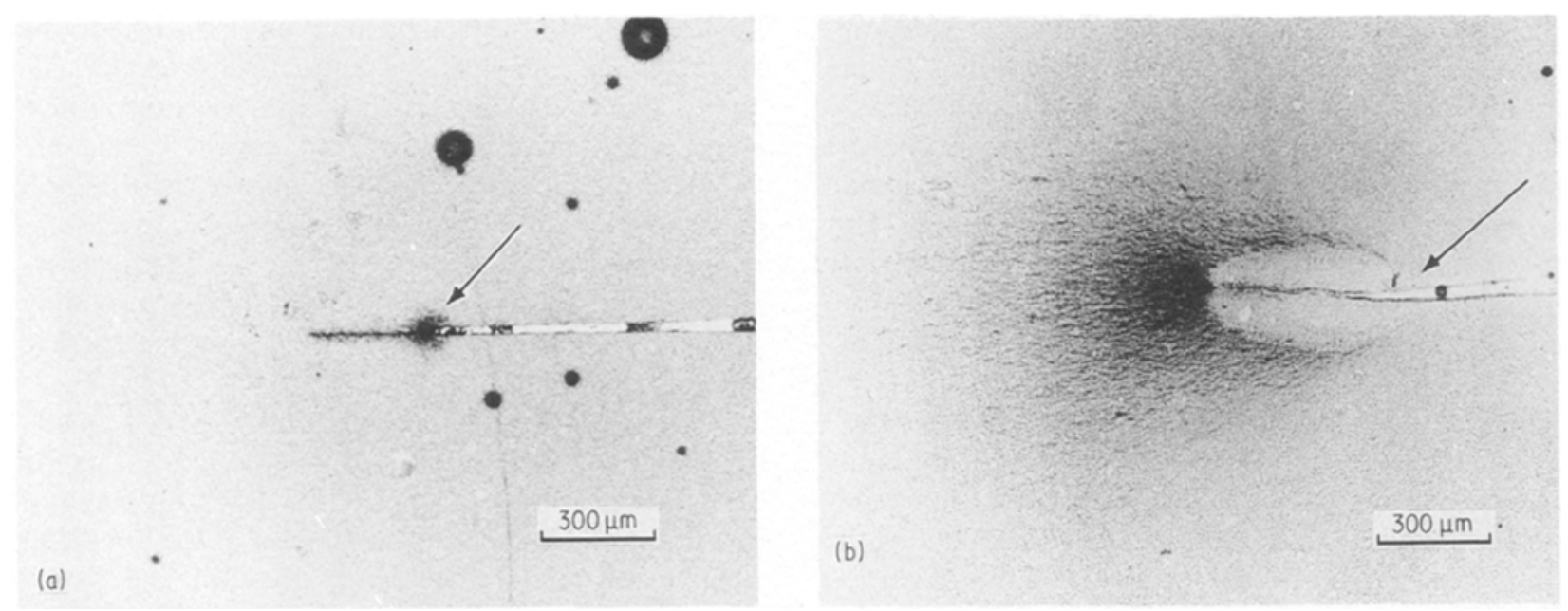

Figure 7 Optical micrographs of the crack tip damage zone of PA/PPO alloy taken in bright field. (a) plane stress region. (b) plane strain region. The arrow indicates the starter crack tip. The crack propagates from right to left.
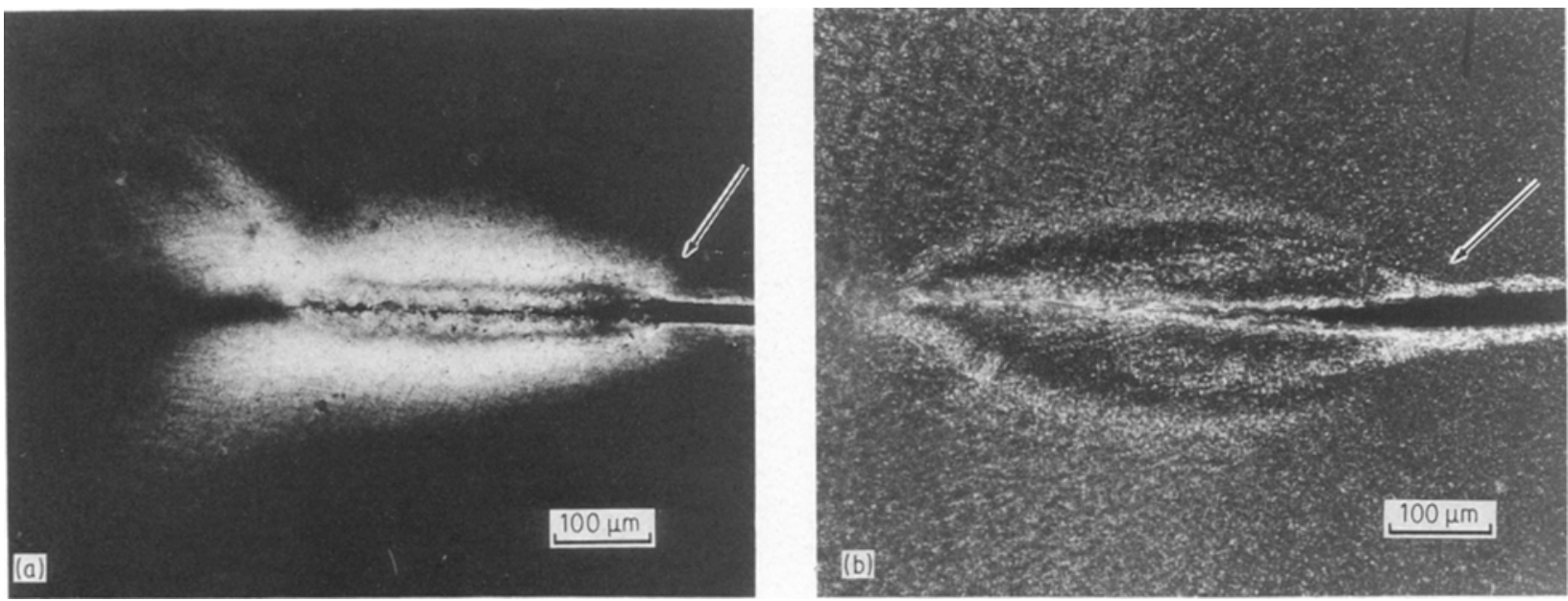

Figure 8 Optical micrographs of the crack tip damage zone of PA/PPO alloy taken in cross-polarized light. (a) plane stress region. (b) plane strain region. The arrow indicates the starter crack tip. The crack propagates from right to left.

rigid* polymer alloys under low temperature and high strain rate conditions. The quantitative contribution of each toughening mechanism in the PA/PPO alloy will not be discussed in this paper. Nevertheless, it is believed that the size of the damage zone corresponds qualitatively to its toughness if the major operative toughening mechanisms in the damage zone are the same. Further, any observation of the existing toughening mechanism(s) under low temperature and high testing rate conditions will help provide clues for selecting the correct parameters for optimizing the toughness of the system under a given set of testing conditions. Therefore, understanding of all the available toughening mechanisms that can operate in the material under the given conditions is extremely important for material optimization.

The previous paper [5] reveals that the toughening mechanisms in the PA/PPO alloy are the formation of the crazing zone, followed by shear plastic flow inside the crazed zone. The testing condition was held at a slow rate $\left(8.47 \mathrm{~mm} \mathrm{~s}^{-1}\right)$ and at room temperature. The present work extends the investigation to the fast fracture region, i.e., the sample is tested under the same slow rate and room temperature conditions. In the fast fracture region, the formation of crazes in the SFSZ is interesting though not expected (based on the optical microscopy observations shown in Fig. 4). This phenomenon indicates that the crazing mechanism can be operative in the PA/PPO alloy even when the crack propagates with a relatively high speed in the fast fracture region. Consequently, it is conceivable that if one can modify the PA/PPO in such a way that the crazing mechanism is further promoted, then, the modified PA/PPO alloy can become more resistant to fast fracture propagation. This may, in turn, improve the toughness of the PA/PPO alloy at impact rates and low temperatures, so long as the failure mechanisms in the fast fracture region of the sample tested at room temperature and low rates are similar to that in the impact-tested specimen. This leads us to study the failure mechanisms of the impact-tested PA/PPO.

The fact that the PPO particles stay approximately

* In the context of this paper rigid means non-elastomeric. 
spherical in the SFSZ except on the crack face indicates that very little, if any, shear yielding occurs around the crack tip when the crack propagates. The major energy dissipation mechanisms in the fast fracture region are probably the crack bridging mechanism and the limited formation of crazes. This observation is consistent with a notion we previously proposed $[3,7]$ that the shear plastic flow mechanism and the crack bridging mechanism are mutually exclusive.

In light of the large elongation of the PPO particles, the adherent strength at the interface must be quite high (Figs 2 and 3). Nevertheless, evidence of debonding at the interface is also observed. Consequently, the ultimate strength of the stretched PPO particles, which have an average of $15 \%$ of the rubbery phase in them, appears to be greater than the interfacial strength between the PA matrix and the PPO particle. Yet, the adherent strength at the interface must be greater than the yield stress of the PPO to cause elongation of the PPO particles. If the adherent strength at the interface is close to the fracture strength of the PPO particles, then maximum toughening by the particle bridging effect could be realized.

The toughening mechanisms in the impact-tested specimen are found to be similar to those in the fast fracture region of the specimen tested at a low rate. Very limited drawing of the PPO particles as well as interfacial failure are found. This indicates that either the interfacial strength is insufficient to sustain the drawing of the PPO particles for an effective crack bridging mechanism or the PPO particles are unable to respond to the impact rate for plastic drawing. The above observations suggest that the degree of interfacial adhesion needed for optimized toughening may be dependent on the testing conditions, i.e., greater interfacial adhesion may be needed for a higher testing rate, provided that all other conditions are the same. In other words, in high rate testing, the yielding stress of the PPO may have been raised to a value close to the cohesive strength of the PPO particles. The PPO particles have been fractured before they are able to effect the particle bridging mechanism.

When the PA/PPO specimen is equilibrated in the liquid nitrogen bath and then impact tested, the toughening mechanism in this alloy in the plane strain region is found to be mainly transparticle fracture. In this test, the specimen is at a temperature below the $T_{\mathrm{g}}$ of the rubber $\left(\approx-80^{\circ} \mathrm{C}\right)$. As a result, the elastic constants among the PA, the PPO, and the rubber should be very close. The stress concentration due to the dispersed particles should become minimal. Consequently the crack propagates without being deflected by the particles. Only when interfacial adherent failure occurs does the crack propagate along the interface. Furthermore, the energy dissipation mechanism is limited to the creation of the the new surface and what viscous processes there are near the surface. No other significant dissipation mechanisms are apparent.

The previous [5] and present studies on the PA/PPO system indicate that, depending on the test- ing conditions, the toughening mechanisms of the PA/PPO alloy may change from crazing/shear yielding to crack bridging/crazing, and to transparticle failure. The present study also suggests that the PA/PPO can undergo limited plastic deformation under impact conditions. Thus, it may be possible to improve the impact property of the PA/PPO by activating more SFSZ craze formation and by providing stronger interfacial adhesion to optimize the particle bridging efficiency.

Since the shear yielding mechanisms can, at most, be operative immediately around the crack tip, the cavitational process in front of the crack tip may not be critical to the fracture toughness of the PA/PPO when tested under high rates. Instead, one would probably prefer to toughen the impact property (high testing rate) of the PA/PPO system with multiple crazing and particle bridging mechanisms. As a result, parameters such as interfacial adhesion, particle size and their distribution, mechanical properties of the dispersed phase, and particle/rubber concentration may become more critical to the impact property of the PA/PPO alloy $[6,7,11,19-22]$.

The above observation appears to contradict a recent work conducted by Hobbs and Dekkers [10]. They suggest that no crazing and cavitation occurs in the PA matrix when the PA/PPO alloy is tested under both tension and Izod impact conditions. In addition, they inferred from volume dilatometry experiments that the failure mechanisms at low and high deformation rates are similar. Our previous and current investigations have produced direct evidences that disagree with their conclusions. The crazes are indeed formed from inside the PPO particle and extend to the PA matrix (Fig. 9). The reason why they did not observe the crazes in the PA matrix may be mainly because they either used SEM (which is difficult to use for identifying crazing) or TEM viewing in an inappropriate location (crazes only form immediately beneath the crack surface of the core region).

In order to further support the above viewpoint, the damage zones both in the skin (plane stress) region

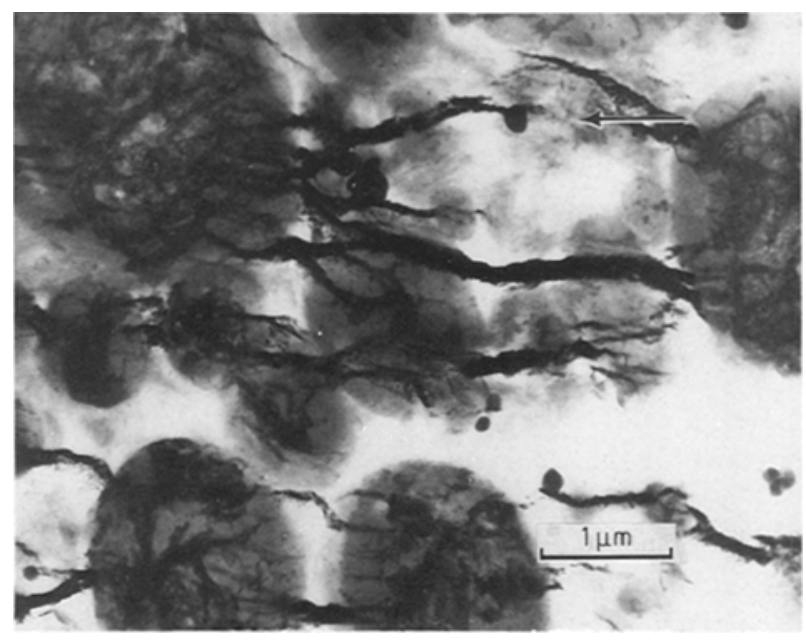

Figure 9 A TEM micrograph of a SEN-3PB thin section obtained from the SFSZ. The crazes appear to initiate from the elastomer phase inside the PPO. The arrow indicates the crack propagation direction. 


\begin{tabular}{ll}
\hline Testing condition/Location of investigation & $\begin{array}{l}\text { Major failure mechanisms } \\
\text { (" } \rightarrow \text { ": indicates the sequence of events) }\end{array}$ \\
\hline $25^{\circ} \mathrm{C}, 8.47 \mathrm{~mm} \mathrm{~s}^{-}, 6.35 \mathrm{~mm}$ thick bar. (Stress-whitened zone) & Crazing $\rightarrow$ Shear yielding \\
Core & Shear yiclding $\rightarrow$ Rubber cavitation [10] \\
Skin & Particle bridging; Crazing \\
$25^{\circ} \mathrm{C}, 8.47 \mathrm{~mm} \mathrm{~s}^{-1}, 6.35 \mathrm{~mm}$ thick bar. (Fast fracture region) & \\
Core & Particle bridging; Crazing \\
$25^{\circ} \mathrm{C}, 4 \mathrm{~m} \mathrm{~s}^{-1} 6.35 \mathrm{~mm}$ thick bar. & Transparticle fracture \\
$\approx-150^{\circ} \mathrm{C}, 4 \mathrm{~m} \mathrm{~s}^{-1} 6.35 \mathrm{~mm}$ thick bar. & Tore
\end{tabular}

and the core (plane strain) region are investigated. Indeed, using bright field conditions (Fig. 7), the crazes are not observed when the investigation is conducted at the specimen surface. However, when the plane strain core region is studied, the crazing mechanism is present around the crack tip. Interestingly, when these damage zones are viewed under cross-polarized light, the size of the birefringent shear yielded zones of both the surface and core regions are similar (Fig. 8). This finding implies that the cavitational process, which is in the form of massive crazing around the crack tip, not only relieves the constraint induced by the thickness of the sample, but also further activates the shear yielding process. The effect of the crack tip cavitational process in polymer toughening will be discussed in a separate paper.

The evidence that the failure mechanisms between the plane stress and plane strain regions in the PA/PPO alloy are different does indicate that the specimen geometry and testing conditions will greatly alter the failure process, and thus, the toughening strategy. Dekkers et al. $[10,15]$ and Guild and Young [23] proposed that the stress concentration build-up at the interface due to the discrepancy of their elastic moduli is the main cause for the localized shear banding around the propagated crack. However, based on our finite element methods simulation and micromechanics analyses $[7,24,25]$, along with the current finding, it is clear that only when the specimen is tested under uniaxial or biaxial tension, or that the specimen is relatively thin and the crack is relatively blunt, is the stress concentration effect sufficient for causing localized shear banding, thereby possibly toughening the system. On the other hand, if the specimen is thick and the material experiences a triaxial tension, e.g., in front of a sharp crack, then, the constraint in front of the crack tip needs to be relieved [2]. The stress concentration effect alone is not sufficient for inducing massive shear plastic flow around the crack tip.

In the case of the plane stress condition, where the fracture mechanisms of materials on the specimen surface are studied (Figs. 7a and 8a), a small spherical cavitation zone is found directly in front of the starter crack tip. This cavitation zone is far smaller than the crazed zone found inside the core region. This zone is also slightly smaller than the skin shear yielded region immediately ahead of the starter crack tip. This finding implies that the cavitation zone could be formed after the formation of the shear yielded zone. Consequently, the cavitational process in the plane stress condition could be a result of the crack tip shear yielding process. A similar work conducted by Hobbs and Dekkers [10] also found the evidence of SFSZ cavitation in the skin region of the specimen. They pointed out that the cavitational process is due to the rubbery phase cavitation. No crazing mechanism was observed. Their work is, in fact, in agreement with the present observations, if the stress state that the material experiences is taken into account. For clarity, the failure mechanisms in PA/PPO under various testing conditions are summarized in Table I.

In an experiment using a tapered tensile specimen conducted previously [5], we found that under uniaxial tension conditions the crazing mechanism is suppressed. Not until the material reaches the bulk yielding of the specimen could the crazing mechanism be resolved (Fig. 10). This finding indicates that under the plane stress condition, the triaxial tension is too low to effectively activate the crazing mechanism in PA/PPO alloy. Further, a recent work conducted by Pearson and Yee [26] on PPO modified epoxy system shows that without any addition of the elastomeric phase, massive localized shear banding mechanism can still occur in simple tension. The above evidence strongly suggests that the cavitational process under the plane stress condition is not critical for the initiation of the shear yielding mechanism.

By analyzing the contradictions in the literature concerning the sequence of toughening events and the cause for the crack tip shear yielded zone $[2,5,10$, 14-17], it is now apparent that these inconsistencies are mainly due to different locations of the specimen and the use of inadequate tools in their analyses, i.e., analyses were made at different stress states and testing conditions. For example, Guild and Young [23] concluded that cavitation of rubber particles is not essential prior to yielding. They used the finite element methods to study the stress field around the rubber particle under uniaxial tension condition. In reality, the hydrostatic tension around the rubber particle in front of a sharp crack tip is much greater than that in the uniaxial tension case $[7,25]$. Their conclusion can only be correct when the yielding stress of the matrix is 


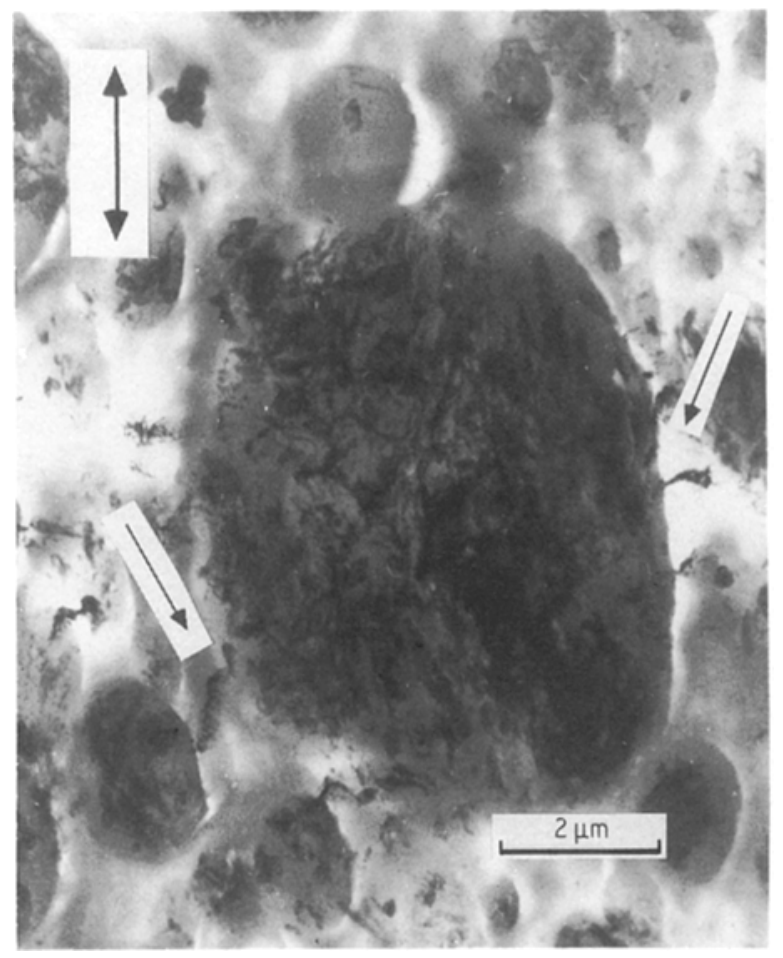

Figure 10 A TEM micrograph of the tested tapered tensile specimen. The micrograph shows evidence of craze formation (see arrows) in the stress-whitened zone. These crazes are rather small, compared to the crazes in Fig. 9. Note that the PPO particles have been deformed along the tensile direction as indicated by the double arrow.

far lower than its brittle stress for failure, such as polyethylene and polybutene-1 [1].

\section{Conclusion}

Toughening mechanisms of a PA/PPO alloy tested at a slow rate, an impact rate, and at a low temperature have been investigated using SEM, TOM, and TEM. The toughening mechanisms may change from crazing and shear yielding to crack bridging/crazing, and to transparticle failure, depending on the testing conditions. Since multiple crazing and particle bridging mechanisms appear to dominate the toughening of $\mathrm{PA} / \mathrm{PPO}$ at high testing rates, one would probably prefer to improve the impact property of the PA/PPO alloy with multiple crazing and particle bridging mechanisms. As a result, parameters like interfacial adhesion, particle size and their distribution, and rubber concentration may become more critical to the impact property of the PA/PPO alloy.

\section{Acknowledgement}

This work is supported by a Material Research Group grant from the National Science Foundation, No. DMR-8708405. The authors would like to thank Lisa Maison for help with the TEM work.

\section{Reference}

1. I. M. WARD, "Mechanical Properties of Solid Polymers", 2nd ed. (Wiley \& Sons, New York, 1983).

2. (a) A. F. YEE and R. A. PEARSON, J. Mater. Sci., 21 (1986) 2462; (b) Idem. ibid. 21 (1986) 2475.

3. D. S. PARKER, H. J. SUE, J. HUANG, and A. F. YEE, Polymer, 31 (1990) 2267.

4. A. F. YEE, R. A. PEARSON, and H. J. SUE, "Fracture and Toughening Mechanisms in Polymers", 7th International Conference on. Fracture 4 (1989) 2739.

5. H. J. SUE and A. F. YEE, J. Mater. Sci. 24 (1989) 1447

6. A. C. GARG and Y. W. MAI, Composites Science and Technology 31 (1988) 179.

7. Idem, "Toughening Mechanisms in Alloys of Rigid Polymers", 7th International Conference on Deformation, Yield and Fracture of Polymers (Cambridge, England, April 1988).

8. H. J. SUE, Polym. Eng. Sci., 31 (1991) 275.

9. Idem, Ph.D. Thesis (The University of Michigan, Ann Arbor, MI, 1988).

10. S. Y. HOBBS and M. E. J. DEKKERS, J. Mater. Sci. 24 (1989) 1316.

11. S. KUNZ-DOUGLASS, P. W. R. BEAUMONT and M. F ASHBY, J. Mater. Sci. 16 (1981) 2657.

12. A. J. KINLOCH, S. J. SHAW, D. A. TOD, and D. L. HINSTON, Polymer 24 (1983) 1341.

13. H. J. SUE, R. A. PEARSON, D. S. PARKER, J. HUANG, and A. F. YEE, Polymer Preprint 29 (1988) 147

14. A. J. KINLOCH, "Mechanics and Mechanisms of Fracture of Thermosetting epoxy Polymers", in "Advances in Polymer Science", Vol. 72, edited by K. Dusek (Springer-Verlag, Berlin, 1986). p. 45 .

15. M. E. J. DEKKERS, S. Y. HOBBS, V. H. WATKINS, J. Mater. Sci. 23 (1988) 1225.

16. T. K URA UCHI and T. OHTA, J. Mater. Sci. 19 (1984) 1699.

17. A. MARGOLINA and S. WU, Polymer 29 (1988) 2170.

18. A. S. HOLIK, R. P. KAMBOUR, S. Y. HOBBS, and D. G. FINK, Microstruct. Sci. 7 (1979) 357.

19. A. J. KINLOCH and J. G. WILliaMS, J. Mater. Sci. 15 (1980) 987.

20. R. O. RITCHIE and W. YU, 'Short Crack Effects in Fatigue: A Consequence of Crack Tip Shielding' in "Small Fatigue Cracks", edited by R. O. Ritchie and J. Lankford (TMS-AIME, Warrendale, PA, 1986).

21. J. G. WILliams and J. M. HOdGKINSON, Proc. Roy. Soc. A375 (1981) 231

22. C. B. BUCKNALL, "Toughened Plastics" (Applied Science, London, 1977).

23. F. J. GUILD and R. J. YOUNG, J. Mater. Sci. 24 (1989) 2454

24. H. J. SUE and A. F. YEE, Polymer 29 (1988) 1619.

25. F. LI, A. F. YEE, and J, PAN, to be published.

26. R. A. PEARSON and A. F. YEE, to be published.

\section{Received 11 June}

and accepted 26 June 1990 